\title{
Thermodynamic admissibility of Bouc-Wen type hysteresis models
}

\author{
Silvano Erlicher ${ }^{\text {a,b }}$, Nelly Point ${ }^{\text {a,c }}$ \\ a Laboratoire d'Analyse des Matériaux et Identification LAMI (ENPC/LCPC-Institut Navier), 6 et 8 avenue \\ Blaise Pascal, Cité Descartes, Champs-sur-Marne, 77455 Marne-la-Vallée, Cedex2, France \\ E-mail: erlicher@lami.enpc.fr Tel: 0033 - (0)1 64153740 \\ E-mail: point@lami.enpc.fr Tel: 0033 - (0)1 64153740 \\ Fax: 0033 - (0)1 64153741 \\ b Università di Trento, Dipartimento di Ingegneria Meccanica e Strutturale (DIMS), Via Mesiano 77, 38050, \\ Trento, Italy \\ E-mail: silvano.erlicher@ing.unitn.it \\ Tel: 0039 - 0461882546 Fax: 0039 - 0461882505 \\ c Conservatoire National des Arts et Métiers, Département de Mathématiques, 292 rue Saint-Martin, 77141 \\ Paris, Cedex03, France \\ E-mail: point@cnam.fr \\ Tel: 0033 - (0)1 $40272543 \quad$ Fax: 0033 - (0)1 40272746
}

(Reçu le jour mois année, accepté après révision le jour mois année)

Abstract. Starting from the relationship between the Bouc model and the endochronic theory and by adopting some new intrinsic time measures, the thermodynamic admissibility of the BoucWen model is proved, in the univariate case as well as in the tensorial one. Moreover, the proposed proof encompasses the cases where a strength degradation term appears. (C) 2001 Académie des sciences/Éditions scientifiques et médicales Elsevier SAS

Thermodynamics / Hysteresis / Bouc-Wen models / Endochronic theory / Seismic engineering

Admissibilité thermodynamique de modèles d'hystérésis de type Bouc-Wen

Résumé. Partant de la relation entre le modèle de Bouc et la théorie endochronique et grâce à l'introduction de nouveaux temps internes, l'admissibilité thermodynamique du modèle de BoucWen est prouvée, aussi bien dans le cas scalaire que dans le cas tensoriel. De plus, la preuve proposée s'applique également s'il y a un terme prenant en compte la dégradation de la force maximale. (c) 2001 Académie des sciences/Éditions scientifiques et médicales Elsevier SAS

Thermodynamique / Hystérésis / Modèles de Bouc-Wen / Théorie endochronique / Ingénierie sismique

Note présentée par Pierre SUQUET

S1620-7742(01)0????-?/FLA

(c) 2001 Académie des sciences/Éditions scientifiques et médicales Elsevier SAS. Tous droits réservés. 


\section{S. Erlicher, N.Point}

\section{Introduction}

Among the smooth univariate hysteresis models, the Bouc model [1] and the Bouc-Wen model [2] are the most popular ones in structural dynamics. They are employed, in particular, in seismic engineering as analytical tools to represent the cyclic behaviour of structural members, structural joints and isolation devices (see, among others, [3] and [4]). However, it is often argued that these models do not respect the Drucker's postulate [5], while the study of their thermodynamic admissibility is faced only for some particular parameter choices [6] [7] [8]. The aim of this note is to prove the thermodynamic admissibility of the Bouc-Wen model in a more general framework, using a tensorial formulation and taking into account the so-called strength degradation effect [9]. Our approach makes use of ideas introduced by Valanis [10] in his endochronic theory of plasticity. The proposed proof is based on the choice of suitable intrinsic time scales, more general than the ones usually adopted in the standard endochronic theory.

\section{Bouc and Bouc-Wen type models}

Among the different univariate models of hysteresis proposed by Bouc [1], the simplest one is :

$$
\left\{\begin{array}{l}
w(t)=A_{0} u(t)+z(t) \\
z(t)=\int_{0}^{\vartheta(t)} \mu\left(\vartheta(t)-\vartheta^{\prime}\right) \frac{d u}{d \vartheta^{\prime}} d \vartheta^{\prime}
\end{array}\right.
$$

where $u$ and $w$ are two time-dependent functions, with the role of input and output respectively. Moreover, $A_{0} \geqslant 0$ and $\mu=\mu(\vartheta)$ is the so-called hereditary kernel. The time function $\vartheta$ is positive and nondecreasing, and it is named internal or intrinsic time. One of the definitions of $\vartheta$ proposed by Bouc is the total variation of $u$ :

$$
\vartheta(t)=\int_{0}^{t}\left|\frac{d u}{d \tau}\right| d \tau \quad \text { or, equivalently, } \quad d \vartheta=|d u|, \text { with } \vartheta(0)=0 .
$$

This choice implies the existence almost everywhere of the derivative of the input function $u$ with respect to $\vartheta$ and the rate-independence of $\vartheta$. As a result, $z$ and $w$ are in turn rate-independent.

Bouc defined the hereditary kernel $\mu$ as a continuous, bounded, positive and non-increasing function on the interval $\vartheta \geqslant 0$, having a bounded integral. In particular, the special case of an exponential kernel has been discussed

$$
\mu(\vartheta)=A e^{-\beta \vartheta} \quad \text { with } \quad A, \beta>0
$$

since, under the assumption (2), the following differential formulation of (1) can be deducted

$$
\left\{\begin{array}{l}
w=A_{0} u+z \\
d z=A d u-\beta z|d u|
\end{array}\right.
$$

This is the most popular version of the Bouc model in the civil engineering field and in particular in seismic structural engineering. The input has usually the meaning of a relative displacement between two structural elements, while the output $w$ plays the role of a structural restoring force, defined as the sum of a linear hardening term and a hysteretic term $z$. We observe that the force $z$ is confined to the interval $\left(-z_{u}, z_{u}\right)$, where the limit value $z_{u}$ is equal to $A / \beta$ and represents a maximum strength value of the hysteretic model.

Bouc [11] also proposed a more general formulation of $(4)_{2}$ :

$$
d z=A d u-\beta z|d u|-\gamma|z| d u \quad \text { with } \quad \gamma<\beta
$$

while Wen [2] suggested a further modification introducing the positive exponent $n$ :

$$
d z=A d u-(\beta \operatorname{sign}(z d u)+\gamma)|z|^{n} d u
$$




\section{Thermodynamic admissibility of Bouc-Wen type hysteresis models}

Wen did not impose any condition on the $\gamma$ value and assumed that $n$ is integer. Nevertheless, it is straightforward to prove that all the real positive values of $n$ are admissible. Baber and Wen [9] introduced the so-called stiffness and strength degradation effects in the Bouc-Wen model (6). Only the strength degradation case is considered here:

$$
d z=A d u-\nu(\beta \operatorname{sign}(z d u)+\gamma)|z|^{n} d u
$$

where $\nu$ was defined in [9] as a positive and increasing function of the energy dissipated by the system. Provided that $\beta+\gamma>0$, the strength value $z_{u}$ becomes in this case

$$
z_{u}=\left(\frac{A}{\nu(\beta+\gamma)}\right)^{\frac{1}{n}}
$$

and the degradation effect due to $\nu$ is evident. A tensorial generalization of (6) was suggested by Karray and Bouc [8] for isotropic materials with elastic hydrostatic behaviour:

$$
\left\{\begin{array}{l}
\sigma_{d}=A_{0} \varepsilon_{d}+\mathbf{z} \\
d \mathbf{z}=A d \varepsilon_{d}-\beta \mathbf{z}\|\mathbf{z}\|^{n-2}\left|\mathbf{z}: d \varepsilon_{d}\right|-\gamma \mathbf{z}\|\mathbf{z}\|^{n-2}\left(\mathbf{z}: d \varepsilon_{d}\right)
\end{array}\right.
$$

where $\varepsilon_{d}$ and $\sigma_{d}$ are the deviatoric part of the small strain tensor and of the Cauchy stress tensor, respectively; $\mathbf{z}$ is the tensor defining the hysteretic part of the stress, while $\|\cdot\|$ is the standard $L_{2}-$ norm. Casciati [5] discussed the use of the model (8) in the framework of non-linear stochastic dynamics.

We name Bouc-Wen type models the hysteresis models defined by Eqs. (4) - (8). All of them were originally defined without any thermodynamic analysis. Moreover, the link between the differential formulations of the Bouc-Wen type models (5) - (8) and the original integral formulation (1) due to Bouc, as well as the admissibility intervals for the $\gamma$ parameter, are not discussed in the aforementioned papers. These topics will be studied in the following sections, where it will be proved that both scalar and tensorial Bouc-Wen type models can be formulated within the thermodynamic framework of the endochronic theory.

\section{Thermodynamic principles and thermodynamic potential}

Under the assumptions of isothermal conditions and small transformations, the first principle of thermodynamics and the Helmholtz free energy density can be written as (see, among others, [12]):

$$
\dot{E}=\sigma: \dot{\varepsilon}, \quad \Psi=\Psi\left(\varepsilon, \chi_{1}, \chi_{2}, \ldots, \chi_{N}\right)
$$

where the superposed dot indicates the time derivative; $E$ is the internal energy density; $\varepsilon$ is the small strain tensor; $\sigma$ is the Cauchy stress tensor; while $\chi_{i}, i=1, N$ are internal variables.

The Helmholtz free energy density $\Psi$ has the role of thermodynamic potential. A quadratic convex $\Psi$ function is considered here, depending on the strain tensor $\varepsilon$ and on a single internal variable $\chi$ of tensorial character:

$$
\Psi=\frac{1}{2} \varepsilon: \mathbf{C}: \varepsilon+\varepsilon: \mathbf{B}: \chi+\frac{1}{2} \chi: \mathbf{D}: \chi
$$

where $\mathbf{C}, \mathbf{B}$ and $\mathbf{D}$ are symmetric fourth order tensors. By assuming an isotropic behaviour, one can set:

$$
\mathbf{C}=C_{1} \mathbf{1} \otimes \mathbf{1}+C_{2} \mathbf{I}, \mathbf{B}=B_{1} \mathbf{1} \otimes \mathbf{1}+B_{2} \mathbf{I}, \mathbf{D}=D_{1} \mathbf{1} \otimes \mathbf{1}+D_{2} \mathbf{I}
$$

where $\mathbf{1}$ is the second order identity tensor; $\mathbf{I}$ is the fourth order identity tensor; and $\otimes$ represents the tensor product. The convexity conditions for $\Psi$ can be written as follows:

$$
C_{1}, D_{1}, C_{2}, D_{2}>0, \quad B_{1}^{2} \leqslant C_{1} D_{1}, \quad B_{2}^{2} \leqslant C_{2} D_{2} .
$$




\section{S. Erlicher, N.Point}

In order to have coupling between the deviatoric parts of the strain and the internal variables, it is also assumed $B_{2} \neq 0$. By virtue of (11), the thermodynamic potential (10) becomes:

$$
\Psi=\frac{C_{0}}{2} \operatorname{tr}(\varepsilon)^{2}+\frac{C_{2}}{2} \varepsilon_{d}: \varepsilon_{d}+B_{0} \operatorname{tr}(\varepsilon) \operatorname{tr}(\chi)+B_{2} \varepsilon_{d}: \chi_{d}+\frac{1}{2} D_{0} \operatorname{tr}(\chi)^{2}+\frac{1}{2} D_{2} \chi_{d}: \chi_{d}
$$

where $\varepsilon_{d}$ and $\chi_{d}$ indicate the deviatoric parts of the strain tensor $\varepsilon$ and of the internal variable tensor $\chi$, while $C_{0}=C_{1}+C_{2} / 3, B_{0}=B_{1}+B_{2} / 3$ and $D_{0}=D_{1}+D_{2} / 3$.

The classical expression of the second principle for small isothermal transformations is :

$$
\Phi_{1}(t)=\sigma: \dot{\varepsilon}-\dot{\Psi} \geqslant 0
$$

and it states that the intrinsic (or mechanical) dissipation $\Phi_{1}$ has to be non-negative.

By substituting $(9)_{2}$ in the inequality (14) and knowing that $\Psi$ depends by assumption on a single internal variable, it follows that :

$$
\Phi_{1}(t)=-\frac{\partial \Psi}{\partial \chi} \frac{d \chi}{d t}=-\tau: \dot{\chi} \geqslant 0, \quad \sigma=\frac{\partial \Psi}{\partial \varepsilon}, \quad \tau=\frac{\partial \Psi}{\partial \chi} .
$$

where Eq. (15) 2 is the so-called state equation, while Eq. (15) $)_{3}$ defines the thermodynamic force $\tau$ associated to the internal variable $\chi$. Under the assumption (13), the state equation reads

$$
\sigma=\frac{\operatorname{tr}(\sigma)}{3} \mathbf{1}+\sigma_{d}=\left(C_{0} \operatorname{tr}(\varepsilon)+B_{0} \operatorname{tr}(\chi)\right) \mathbf{1}+C_{2} \varepsilon_{d}+B_{2} \chi_{d}
$$

while the thermodynamic force becomes

$$
\tau=\frac{\operatorname{tr}(\tau)}{3} \mathbf{1}+\tau_{d}=\left(B_{0} \operatorname{tr}(\varepsilon)+D_{0} \operatorname{tr}(\chi)\right) \mathbf{1}+B_{2} \varepsilon_{d}+D_{2} \chi_{d}
$$

The quantities $\tau$ and $\dot{\chi}$ have to be correlated, otherwise a particular evolution of $\chi$ could exist which violates the inequality $(15)_{1}$. Therefore, some additional complementarity rules have to be introduced. A classical method is to assume the existence of a convex positive function $\varphi(\dot{\chi})$, called pseudo-potential, which is zero at the origin and to associate to it a normality condition, namely that the opposite of the thermodynamic force $\tau$ belongs to the sub-differential of $\varphi(\dot{\chi})$ :

$$
\tau \in-\partial \varphi(\dot{\chi})
$$

\section{Intrinsic time and endochronic theory}

Valanis [10] proposed a theory of viscoplasticity without a yield surface, suggesting for it the name of endochronic theory. The basic concept of the Valanis' theory is the existence of the so-called intrinsic time scale or simply intrinsic time, i.e. an ordering variable other than clock-time which governs the behaviour of the material. The intrinsic time $\vartheta \geqslant 0$ is defined as a non-decreasing function, which directly depends on the strain and/or the stress tensors.

The typical definition of the intrinsic time increment is :

$$
d \vartheta=\sqrt{d \varepsilon: \mathbf{p}: d \varepsilon}
$$

where $\mathbf{p}=\mathbf{p}(\sigma, \varepsilon)$ is a positive definite fourth order tensor. Assuming an isotropic behaviour, the hydrostatic and the deviatoric responses can be uncoupled, leading to intrinsic time definitions as, for instance, the following one :

$$
d \vartheta=\sqrt{d \varepsilon_{d}: \mathbf{p}: d \varepsilon_{d}} \quad \text { with } \mathbf{p}=\mathbf{p}\left(\sigma_{d}, \varepsilon_{d}\right) \text { positive definite }
$$




\section{Thermodynamic admissibility of Bouc-Wen type hysteresis models}

where $\vartheta$ depends only on deviatoric tensors. In both cases, the intrinsic time definitions possess the rateindependence property. $\vartheta$ :

According to the Valanis' formulation, the second principle inequality $(15)_{1}$ is rewritten by introducing

$$
\Phi_{1}(t)=-\tau: \frac{d \chi}{d \vartheta} \frac{d \vartheta}{d t}=-\tau: \hat{\chi} \frac{d \vartheta}{d t} \geqslant 0
$$

The non-negativity of $d \vartheta$ has been imposed by definition. Therefore, in order to satisfy the inequality (20), it is sufficient to assume the existence of a positive convex dissipation potential $\varphi=\varphi(\hat{\chi})$, with $\hat{\chi}=\frac{d \chi}{d \vartheta}$, and a normality condition :

$$
\frac{d \vartheta}{d t} \geqslant 0, \quad \varphi=\varphi(\hat{\chi}) \geqslant 0, \quad \varphi(\mathbf{0})=0, \quad \tau \in-\partial \varphi(\hat{\chi}) .
$$

The hypotheses (21) have the role of complementarity rules for the endochronic theory, in the sense that they are sufficient to ensure that the second principle is fulfilled.

Assuming that the hydrostatic parts of $\sigma$ and $\tau$ have an elastic behaviour, a dissipation potential depending quadratically on the deviatoric part of $\hat{\chi}$ is considered :

$$
\varphi=\varphi(\hat{\chi})=I_{0}(\operatorname{tr}(\hat{\chi}))+\frac{b_{2}}{2}\left\|\hat{\chi}_{d}\right\|^{2} \quad \text { with } b_{2}>0 \text { and } I_{0}(\operatorname{tr}(\hat{\chi}))= \begin{cases}0 & \text { if } \operatorname{tr}(\hat{\chi})=0 \\ \infty & \text { elsewhere }\end{cases}
$$

where $I_{0}(\operatorname{tr}(\hat{\chi}))$ is the indicator function of the set $\mathbb{E}=\{\hat{\chi} \mid \operatorname{tr}(\hat{\chi})=0\}$.

We should emphasize that this pseudo-potential is rate independent, even though it is not a homogeneous function of order 1, as in classical plasticity. The rate-independence is ensured by the use of the internal time derivative $\hat{\chi}$ instead of $\dot{\chi}$.

The dual dissipation potential $\varphi^{*}$ is obtained by the Legendre-Fenchel transformation of $\varphi$ :

$$
\varphi^{*}(\tau)=\sup _{\hat{\chi}}\left(\tau:(-\hat{\chi})-I_{0}(\operatorname{tr}(\hat{\chi}))-\frac{b_{2}}{2}\left\|\hat{\chi}_{d}\right\|^{2}\right)=\sup _{\hat{\chi}_{d}}\left(\tau_{d}:\left(-\hat{\chi}_{d}\right)-\frac{b_{2}}{2}\left\|\hat{\chi}_{d}\right\|^{2}\right)=\frac{\left\|\tau_{d}\right\|^{2}}{2 b_{2}},
$$

while the dual normality conditions read :

$$
\operatorname{tr}(\hat{\chi})=0, \quad-\hat{\chi}_{d}=\frac{\tau_{d}}{b_{2}} .
$$

The first condition implies that the hydrostatic part of the internal variable remains constant: it is equal to zero if it is assumed that $\operatorname{tr}(\chi(0))=0$. Therefore, referring to (17), one has $\operatorname{tr}(\tau)=3 B_{0} \operatorname{tr}(\varepsilon)$, which describes an elastic hydrostatic response. The linear relation $(24)_{2}$ between $\hat{\chi}_{d}$ and $\tau_{d}$ simply derives from the quadratic form of the deviatoric part of $\varphi^{*}$.

From the deviatoric part of (17) one obtains

$$
\tau_{d}=B_{2} \varepsilon_{d}+D_{2} \chi_{d}
$$

The positivity condition assumed in (19) means that $\varepsilon_{d}$ is continuous as a function of the intrinsic time $\vartheta$ and that its derivative exists almost everywhere. Hence, by deriving (25) and substituting (24) 2 in the obtained expression, the following differential equation is found :

$$
\hat{\tau}_{d}=B_{2} \hat{\varepsilon}_{d}-\frac{D_{2}}{b_{2}} \tau_{d}
$$

If $\tau_{d}(0)=\mathbf{0}$, then the solution of (26) has the form:

$$
\tau_{d}=B_{2} \int_{0}^{\vartheta} e^{-\beta\left(\vartheta-\vartheta^{\prime}\right)} \frac{\partial \varepsilon_{d}\left(\vartheta^{\prime}\right)}{\partial \vartheta^{\prime}} d \vartheta^{\prime} \quad \text { with } \beta=\frac{D_{2}}{b_{2}}>0
$$




\section{S. Erlicher, N.Point}

Hence, by substituting Eqs. (25) and (27) in (16), the following expression for the deviatoric stress tensor is obtained:

$$
\sigma_{d}=\left(C_{2}-\frac{B_{2}^{2}}{D_{2}}\right) \varepsilon_{d}+\frac{B_{2}}{D_{2}} \tau_{d}=\left(C_{2}-\frac{B_{2}^{2}}{D_{2}}\right) \varepsilon_{d}+\frac{B_{2}^{2}}{D_{2}} \int_{0}^{\vartheta} e^{-\beta\left(\vartheta-\vartheta^{\prime}\right)} \frac{\partial \varepsilon_{d}\left(\vartheta^{\prime}\right)}{\partial \vartheta^{\prime}} d \vartheta^{\prime} .
$$

The term proportional to $\varepsilon_{d}$ introduces a linear hardening effect, whereas the integral term corresponds to a smooth hysteretic behaviour, typical of the endochronic theory.

\section{Endochronic theory and Bouc-Wen type models}

In order to investigate the relationship between the endochronic theory and the Bouc-Wen type models, the following parameters are introduced :

$$
A_{0}=C_{2}-\frac{B_{2}^{2}}{D_{2}} \geqslant 0 \quad \text { and } \quad A=\frac{B_{2}^{2}}{D_{2}}>0
$$

The corresponding inequalities are related to the convexity conditions (12). The expression (28) becomes

$$
\sigma_{d}=A_{0} \varepsilon_{d}+\frac{B_{2}}{D_{2}} \tau_{d}=A_{0} \varepsilon_{d}+\int_{0}^{\vartheta} \mu\left(\vartheta-\vartheta^{\prime}\right) \frac{\partial \varepsilon_{d}\left(\vartheta^{\prime}\right)}{\partial \vartheta^{\prime}} d \vartheta^{\prime} \quad \text { with } \mu(\vartheta)=A e^{-\beta \vartheta}
$$

It is the tensorial generalization of (1) for the case of an exponential kernel. Therefore, the previous analysis shows that the exponential form of the hereditary kernel can be determined in a consistent thermodynamic framework. In addition, if $\mathbf{z}$ denotes the integral term in the previous equation, the following differential form of (30) is obtained:

$$
\left\{\begin{array}{l}
\sigma_{d}=A_{0} \varepsilon_{d}+\mathbf{z} \\
d \mathbf{z}=A d \varepsilon_{d}-\beta \mathbf{z} d \vartheta
\end{array}\right.
$$

One can remark that the choice of the internal time increment $d \vartheta$ in Eq. (31) is still open. This additional degree of freedom can be exploited to link the endochronic formulation (31) and the Bouc-Wen type models. The simplest choice $\mathbf{p}=\mathbf{I}$ in (19) leads to $d \vartheta=\left\|d \varepsilon_{d}\right\|$. Hence, by substituting in $(31)_{2}$, one obtains

$$
d \mathbf{z}=A d \varepsilon_{d}-\beta \mathbf{z}\left\|d \varepsilon_{d}\right\|
$$

which is the standard endochronic model [13]. In an univariate structural modelling framework, the changes of variables $\varepsilon_{d} \rightarrow u, \mathbf{z} \rightarrow z$ and $\sigma_{d} \rightarrow w$ can be made and the Bouc formulation (4) $)_{2}$ is found again.

Moreover, the differential formulation $(31)_{2}$ is still valid when the tensor $\mathbf{p}$, which defines the intrinsic time increment $d \vartheta$ according to (19), is positive definite in a non-strict sense. Therefore, the assumption $\mathbf{p}=\mathbf{a} \otimes \mathbf{a}$, with $\mathbf{a}=\mathbf{a}\left(\sigma_{d}, \varepsilon_{d}\right)$ second order tensor different from zero almost everywhere, is admissible. In this case, one has

$$
d \vartheta=\sqrt{d \varepsilon_{d}: \mathbf{a} \otimes \mathbf{a}: d \varepsilon_{d}} \quad \text { or, equivalently, } \quad d \tilde{\vartheta}:=\mathbf{a}: d \varepsilon_{d}, \quad d \vartheta:=|d \tilde{\vartheta}|
$$

where $d \vartheta$ is zero not only for $d \varepsilon_{d}=\mathbf{0}$, but also when $d \varepsilon_{d} \perp \mathbf{a}$ and $\mathbf{a}=\mathbf{0}$. The definition (33) can be generalized as follows:

$$
\begin{array}{ll}
d \tilde{\xi}:=\mathbf{a}: d \varepsilon_{d} & \text { with } \mathbf{a}=\mathbf{a} \\
d \vartheta=f(\operatorname{sign}(d \tilde{\xi}))|d \tilde{\xi}| & \text { with } f \geqslant 0
\end{array}
$$

By assuming in (34)

$$
\begin{array}{ll}
d \tilde{\xi}:=\left(\mathbf{z}: d \varepsilon_{d}\right)\|\mathbf{z}\|^{n-2} & \text { with } \mathbf{z}=\sigma_{d}-A_{0} \varepsilon_{d} \\
d \vartheta=f(\operatorname{sign}(d \tilde{\xi}))|d \tilde{\xi}| & \text { with } f=1+\frac{\gamma}{\beta} \operatorname{sign}(d \tilde{\xi}) \geqslant 0
\end{array}
$$


Thermodynamic admissibility of Bouc-Wen type hysteresis models

with $n, \beta>0$, then the following expression of the intrinsic time increment is obtained :

$$
d \vartheta=\left(1+\frac{\gamma}{\beta} \operatorname{sign}\left(\mathbf{z}: d \varepsilon_{d}\right)\right)\left|\mathbf{z}: d \varepsilon_{d}\right|\|\mathbf{z}\|^{n-2} \quad \text { with }-\beta \leqslant \gamma \leqslant \beta
$$

The inequalities involving the $\gamma$ parameter are sufficient to guarantee the non-negativity of $d \vartheta$. On the other hand, it can be proved that they are necessary to fulfil the second principle inequality (14). By substituting the expression (36) in Eq. (31) $)_{2}$, the formulation $(8)_{2}$ of the Karray-Bouc-Casciati model is obtained, which reduces to the Bouc-Wen model (6) in the univariate case. The thermodynamic formulation provided here for these two hysteretic models constitutes a proof of their thermodynamic admissibility, in the sense that they fulfil the second principle inequality. These results extend those concerning the case $\gamma=0$, discussed in [7] for the univariate Bouc model and in [6] for the Karray-Bouc-Casciati model. Moreover, the analysis developed in [8] concerning the case $\gamma=\beta$ is supplemented.

An even more general class of hysteresis models can be defined by extending the intrinsic time definition given in (34):

$$
\begin{array}{ll}
d \tilde{\xi}:=\mathbf{a}: d \varepsilon_{d} & \text { with } \mathbf{a}=\mathbf{a}\left(\sigma_{d}, \varepsilon_{d}\right) \neq \mathbf{0} \text { a.e. } \\
d \vartheta=\nu(\eta) f(\operatorname{sign}(d \tilde{\xi}))|d \tilde{\xi}| & \text { with } f \geqslant 0 \text { and } \nu \geqslant 0 \\
d \eta=f_{1}\left(\sigma_{d}, \varepsilon_{d}\right) d \vartheta & \text { with } f_{1} \geqslant 0
\end{array}
$$

where $\eta$ is an intrinsic time variable governing the strength degradation, while $\nu$ is a positive and increasing function of $\eta$. In particular, one can set

$$
\begin{array}{ll}
d \tilde{\xi}:=\left(\mathbf{z}: d \varepsilon_{d}\right)\|\mathbf{z}\|^{n-2} & \text { with } \mathbf{z}=\sigma_{d}-A_{0} \varepsilon_{d} \\
d \vartheta=\nu(\eta) f(\operatorname{sign}(d \tilde{\xi}))|d \tilde{\xi}| & \text { with } f=1+\frac{\gamma}{\beta} \operatorname{sign}(d \tilde{\xi}) \geqslant 0
\end{array}
$$

and

$$
d \eta:=\mathbf{z}: d \varepsilon^{p}=\frac{\beta \mathbf{z}: \mathbf{z}}{A_{0}+A} d \vartheta=f_{1}\left(\varepsilon_{d}, \sigma_{d}\right) d \vartheta \geqslant 0 \quad \text { with } \quad d \varepsilon^{p}:=d \varepsilon_{d}-\frac{d \sigma_{d}}{A+A_{0}}
$$

In this case the quantity $\eta$ is the dissipated hysteretic energy. With the assumptions (38)-(39) for the intrinsic time increment $d \vartheta$, Eq. (31) $)_{2}$ reads

$$
d \mathbf{z}=A d \varepsilon_{d}-\nu(\eta) \mathbf{z}\left(\mathbf{z}: d \varepsilon_{d}\right)\left(\gamma+\beta \operatorname{sign}\left(\mathbf{z}: d \varepsilon_{d}\right)\right)\|\mathbf{z}\|^{n-2}
$$

which defines a generalized Karray-Bouc-Casciati model with an additional strength degradation term $\nu=\nu(\eta)$. In the univariate case Eq. (7) is retrieved and the thermodynamic admissibility of the related hysteretic model is proved.

\section{Conclusions}

A general proof of thermodynamic admissibility of the Bouc-Wen type models has been proposed, which encompasses the cases where a strength degradation term appears, and provides the intervals of thermodynamically consistent values of the $\gamma$ parameter for both univariate and tensorial models. Work is in progress to extend the proof to the cases with more than one internal variable and with a stiffness degradation term.

\section{References}

[1] R. Bouc, Modèle mathématique d'hystérésis, Acustica 24 (1971) 16-25.

[2] Y.-K. Wen, Method for random vibration of hysteretic systems, J. Eng. Mech Div. ASCE 102 (1976) 249-263.

[3] M.V. Sivaselvan, A.M. Reinhorn, Hysteretic models for deteriorating inelastic structures, J. Eng. Mech. 126-6 (2000) 633-640. 


\section{S. Erlicher, N.Point}

[4] S. Erlicher, Hysteretic degrading models for the low-cycle fatigue behaviour of structural elements: theory, numerical aspects and applications, Ph.D. Thesis, Department of Mechanical and Structural Engineering, University of Trento, Italy, 2003.

[5] F. Casciati, Stochastic dynamics of hysteretic media, Struct. Safety 6 (1989) 259-269.

[6] G. Ahmadi, F.-G. Fan, M.-N. Noori, A thermodynamically consistent model for hysteretic materials, Iranian J. Sci. Tech. 21-3 (1997) 257-278.

[7] D. Capecchi, G. de Felice, Hysteretic systems with internal variables, J. Eng. Mech. 127-9 (2001) 891-898.

[8] M.A. Karray, R. Bouc, Etude dynamique d'un système d'isolation antisismique, Les Annales de 1'ENIT 3-1 (1989) 43-60.

[9] T.T. Baber, Y.-K. Wen, Random vibrations of hysteretic, degrading systems, J. Eng. Mech. Div. ASCE 107-6 (1981) 1069-1087.

[10] K.C. Valanis, A theory of viscoplasticity without a yield surface. Part I: general theory, Arch Mech. 23-4 (1971) 517-533.

[11] R. Bouc, Forced vibrations of a mechanical system with hysteresis, In: Proc. 4th Conf. on Nonlinear Oscillations, Prague, Czechoslovakia, 1967.

[12] O. Coussy, Mechanics of Porous Continua, Wiley, Chichester, 1995.

[13] Z.P. Bazant, Endochronic inelasticity and incremental plasticity, Int. J. Solids Structures 14 (1978) 691-714. 